\title{
TERAPIA COGNITIVO-COMPORTAMENTAL DA FOBIA SOCIAL: MODELOS E TÉCNICAS
}

\author{
Gustavo J. Fonseca D’El Rey \\ Carla Alessandra Pacini
}

\begin{abstract}
RESUMO. A fobia social é um dos transtornos mentais mais prevalentes na população geral. As principais formas de tratamento são a psicoterapia e a farmacoterapia. Este artigo tem como objetivo realizar uma breve revisão bibliográfica dos mais importantes modelos e técnicas cognitivas e comportamentais da fobia social. Os modelos antigos - modelo de déficit de habilidades sociais, modelo de crenças irracionais, modelo da vulnerabilidade cognitiva e os modelos integrativos - serão revisados brevemente. Serão apresentadas as técnicas de tratamento - como a exposição, reestruturação cognitiva, técnicas de relaxamento e treino de habilidades sociais. Estudos de metaanálise sobre a eficácia da terapia cognitivo-comportamental no tratamento da fobia social também serão descritos.
\end{abstract}

Palavras-chave: fobia social, terapia cognitivo-comportamental, tratamento.

\section{COGNITIVE-BEHAVIORAL THERAPY OF SOCIAL PHOBIA: MODELS AND TECHNIQUES}

\begin{abstract}
Social phobia is a widespread mental disorder in the general population. The main forms of treatment are psychotherapy and pharmacotherapy. This article has as objective to carry out a brief bibliographical review of the most important cognitive and behavior models and techniques of social phobia. The old models - social skills deficit model, irrational ideas model, cognitive vulnerability model and the integrative models, will be briefly reviewed. Treatment techniques such as, exposure, cognitive restructuring, relaxation techniques, and social skills training will be presented. Meta-analysis studies about the efficacy of the cognitive-behavioral therapy in the treatment of social phobia will be described.
\end{abstract}

Key words: Social phobia, cognitive-behavioral therapy, treatment.

\section{TERAPIA COGNITIVO-COMPORTAMENTAL DE LA FOBIA SOCIAL: MODELOS Y TÉCNICAS}

RESUMEN. La fobia social es uno de los trastornos mentales más prevalentes en la población general. Las principales formas de tratamiento son la psicoterapia y la farmacoterapia. Este artículo posee como objetivo realizar una breve revisión bibliográfica de los más importantes modelos y técnicas cognitivas y comportamentales de la fobia social. Los modelos antiguos - modelo de déficit de habilidades sociales, modelo de creencias irracionales, modelo de la vulnerabilidad cognitiva y los modelos integrativos - serán revisados brevemente. Serán presentadas las técnicas de tratamiento - como la exposición, reestructuración cognitiva, técnicas de relajamiento y entrenamiento de habilidades sociales. Estudios de metaanálisis sobre la eficacia de la terapia cognitivo-comportamental en el tratamiento de la fobia social también serán descritos.

Palabras-clave: fobia social, terapia cognitivo-comportamental, tratamiento.

* Psicólogo. Especialista. Coordenador e pesquisador do Programa de Fobia Social do Centro de Pesquisas e Tratamento de Transtornos de Ansiedade - São Paulo-SP.

\# Pedagoga. Especialista. Pesquisadora do Programa de Fobia Social do Centro de Pesquisas e Tratamento de Transtornos de Ansiedade - São Paulo-SP. 
A fobia social é considerada um dos transtornos mentais mais prevalentes na população geral (Kessler, McGonagle, Zhao, Nelson, Hughes, Eshleman, Wittchen \& Kendler, 1994; Magee, Eaton, Wittchen, McGonagle \& Kessler, 1996). O termo fobia social ou transtorno de ansiedade social é usado para a ansiedade intensa em situações sociais e de desempenho, que leva ao sofrimento e perdas de oportunidades (D'El Rey, 2001).

Segundo o DSM-IV-TR (APA, 2002), a fobia social é caracterizada por um medo acentuado e persistente de uma ou mais situações sociais ou de desempenho. A pessoa teme agir de um modo ou mostrar sintomas de ansiedade que lhe sejam humilhantes e embaraçosos, e a exposição à situação social temida provoca uma resposta de ansiedade intensa, que pode chegar a um ataque de pânico. A pessoa geralmente evita estas situações ou as suporta com intenso sofrimento. A fobia social apresenta significativa interferência nas rotinas de trabalho, acadêmicas e sociais e/ou sofrimento acentuado por ter a fobia.

O medo social na grande maioria das vezes está associado às situações de desempenho, como falar em público, as interações sociais do dia-a-dia, como ir a uma festa, uma entrevista de emprego, etc. (Furmark, Tillfors, Stattin, Ekselius \& Fredrikson, 2000; Stein, Torgrud \& Walker, 2000). As pessoas diagnosticadas como fóbicas sociais apresentam uma hipersensibilidade a criticas, mantêm uma avaliação negativa a respeito de si mesma, sentimentos de inferioridade, e apresentam grande dificuldade em serem assertivas (Lamberg, 1998; Stopa \& Clark, 1993).

\section{MÉTODO}

Realizamos uma busca bibliográfica pela internet através do Medline, Psycinfo e do Lilacs (todos os anos disponíveis), com as palavras-chave: social phobia, social anxiety disorder, cognitive-behavioral therapy, psychotherapy, treatment, models, exposure, cognitive restructuring, relaxation techniques, social skills training, meta-analysis. Procuramos incluir os artigos mais recentes em cada subtópico do presente trabalho, embora alguns artigos mais antigos (década de 70 e 80) tenham sido incluídos devido a sua relevância no subtópico.

\section{PRINCIPAIS MODELOS COGNITIVOS E COMPORTAMENTAIS DA FOBIA SOCIAL}

\section{Modelos antigos}

Modelos mais antigos de explicação da fobia social se focalizaram no condicionamento clássico e postularam que uma experiência traumática, como um embaraço momentâneo em uma situação social, poderia ser responsável pelo início da fobia (Öst \& Hugdahl, 1981) - por exemplo, um aluno ficar muito nervoso ao ter que ler um texto diante dos demais alunos em sala de aula. Este fato poderia fazer com que o referido aluno não mais quisesse ler/falar em público. Hofmann, Ehlers e Roth (1995) relataram que estas experiências traumáticas têm sido reportadas por pessoas com ansiedade severa ao falar em público, porém em quase todos os casos ela ocorreu depois que os sintomas fóbicos sociais já eram proeminentes na pessoa. Devido a isto, pode-se dizer que tais experiências negativas eram já uma consequiência da fobia, e não o início do transtorno.

\section{Déficit de habilidades sociais}

Esta teoria postula que indivíduos com fobia social sofrem de um grande déficit nas habilidades sociais, como, por exemplo, iniciar uma conversa com uma pessoa estranha, comportar-se em uma entrevista de emprego, comportamentos de paquera, etc. Deficiências nas habilidades sociais podem levar uma pessoa a se comportar de uma forma inadequada em um primeiro encontro romântico e isto deixá-la em uma situação embaraçosa, por exemplo. A falta de habilidade social pode levar o indivíduo a evitar situações sociais onde ela (habilidade) possa ser essencial para um comportamento adequado e efetivo, como uma entrevista de emprego. Os estudos relatam que existe uma alta incidência de déficits de habilidades sociais em pacientes com diagnóstico de fobia social, e que este fato seria pelo menos responsável pela manutenção do quadro fóbico (Mersch, 1995; Öst, Jerremalm \& Johansson, 1981).

\section{Crenças irracionais}

Para Ellis (1962), as crenças irracionais são o centro dos transtornos neuróticos. Esse autor teorizou que a ansiedade social poderia ser explicada pelas crenças irracionais que a pessoa tem - por exemplo, a crença de que temos sempre que causar uma boa impressão para sermos aceitos pelas pessoas. Outro tipo de crença irracional é a de que temos sempre que ter um desempenho perfeito em determinada situação para mostrarmos o nosso valor como pessoa. Como conseqüência, as pessoas que mantêm estes tipos de crença tendem a se cobrar demais, querendo sempre ter um desempenho perfeito, sem erros, chegando a uma tentativa extrema de perfeição. Este alto grau de exigência leva a um alto nível de ansiedade na execução do comportamento e de frustração quando algo não sai como planejado pelo indivíduo. 


\section{Vulnerabilidade cognitiva}

Beck, Emery e Greenberg (1985) teorizaram que existe uma vulnerabilidade cognitiva nos pacientes com fobia social, ou seja, estes pacientes tendem a interpretar de forma errônea as situações sociais e seu próprio desempenho.

Conforme Beck, Emery e Greenberg (1985); Stopa e Clark (1993); Taylor, Woody, Koch, McLean, Patterson e Anderson (1997), o medo central na fobia social é o de ser o foco das atenções, de expor suas fraquezas e em conseqüência disto ter seu desempenho avaliado de forma desfavorável.

Ainda conforme estes mesmos autores, o fóbico social apresenta falhas no processamento cognitivo, que tende a distorcer a avaliação de suas experiências interpessoais. Ele mantém pensamentos patologicamente negativos acerca de si mesmo, suas experiências e seu futuro; seletivamente procura evidências para reafirmar sua visão negativa, reforçando suas crenças e mantendo os sintomas cognitivos, comportamentais e fisiológicos. Os erros sistemáticos do processamento cognitivo levam à manutenção dos pensamentos distorcidos do paciente, apesar das evidências em contrário.

\section{Modelos Integrativos}

\section{Modelo cognitivo-comportamental da fobia social}

O modelo cognitivo-comportamental proposto por Heimberg, Juster, Hope e Mattia (1995) procura integrar vários resultados de pesquisas e modelos existentes. $\mathrm{O}$ modelo é baseado na presunção de que existe uma predisposição para o desenvolvimento da fobia social, que pode ser herdada ou produzida por fatores na infância ou na adolescência que sensibilizam o indivíduo para os aspectos ameaçadores das situações sociais. Tais fatores podem incluir pais com ansiedade social, padrões exagerados de perfeição no funcionamento, superproteção e/ou isolamento dos contatos sociais. Estes mesmos autores, indicam que estes fatores vividos na infância ou adolescência aumentam a probabilidade de a pessoa entrar em situações sociais de forma apreensiva, desta maneira sentindo muita ansiedade ou tentando evitá-las. Estas pessoas formam cognições de que as situações sociais são perigosas e que a única maneira de prevenir resultados negativos é evitá-las. Conseqüentemente tendem a antecipar uma possível humilhação e embaraço, e devido a isto evitam as situações sociais ou de desempenho; e quando não conseguem evitá-las sofrem em demasia antes e durante a situação.

\section{Modelo cognitivo da fobia social}

Clark e Wells (1995) propõem um modelo cognitivo para a fobia social. Neste modelo, o fóbico social é motivado a interpretar as situações sociais de forma negativa e conseqüentemente sentir-se inseguro e/ou evitar estas situações. Estas pessoas percebem as situações sociais e de desempenho como extremamente ameaçadoras. Esta distorção da realidade resultaria de pensamentos denominados de automáticos, pois surgem rapidamente e sem motivo real, desencadeando a ansiedade social.

Segundo Clark e Wells (1995); Stopa e Clark (1993), os pacientes com fobia social são fortemente predispostos a sentir que os outros os avaliam de forma negativa. Esses pensamentos distorcidos provocam ansiedade e muitas vezes atrapalham a realização do comportamento social, gerando desta maneira um circulo vicioso. Também apresentam expectativas nãorealistas sobre suas habilidades para lidar com situações sociais, superestimam a probabilidade de ocorrerem eventos sociais negativos e sentem-se constantemente criticados ou desaprovados. Similarmente, Beck, Emery e Greenberg (1985); Lucock e Salkovskis (1988), propõem a existência de uma falha no processamento da informação nos indivíduos com fobia social, pois eles interpretam as situações sociais como mais ameaçadoras do que realmente o são.

\section{TRATAMENTO DA FOBIA SOCIAL}

Atualmente os pesquisadores e clínicos têm focalizado sua atenção sobre o tratamento com a terapia cognitivo-comportamental e a farmacoterapia na fobia social (Liebowitz, 1999). Os inibidores seletivos de recaptação de serotonina vêm constituindo-se como uma das principais intervenções farmacológicas na fobia social (Schneider, 1995). Em relação à terapia cognitivocomportamental da fobia social, as principais técnicas que vêm sendo pesquisadas são a exposição, reestruturação cognitiva, técnicas de relaxamento e treino de habilidades sociais (Heimberg, 2001; Lincoln, Rief, Hahlweg, Frank, Schroeber \& Fiegenbaum, 2003). Muitas destas técnicas cognitivas e comportamentais derivam de modelos psicológicos e biológicos dos transtornos de ansiedade.

\section{TÉCNICAS COGNITIVAS E COMPORTAMETAIS}

\section{Técnicas de relaxamento}

As técnicas de relaxamento ajudam o paciente a controlar sintomas fisiológicos antes ou durante os eventos temidos. A principal técnica de relaxamento 
utilizada é o Relaxamento Progressivo de Jacobson. Öst, Jerremalm e Johansson (1981) sugerem que as técnicas de relaxamento são apropriadas a pacientes com fobia social que apresentam intensos sintomas fisiológicos de ansiedade durante uma situação social ou de desempenho. Os estudos mostram que as técnicas de relaxamento facilitam a exposição do paciente à situação temida, porém não são eficazes isoladamente no tratamento da fobia social (Jerremalm, Jansson \& Öst, 1986; Öst, Jerremalm \& Johansson, 1981; Otto, 1999).

\section{Treinamento de habilidades sociais}

Segundo Argyle, Bryant e Trower (1974), as mais comuns técnicas usadas no treinamento de habilidades sociais são a modelagem pelo terapeuta, ensaio comportamental, reforçamento social e o treinamento realizado fora da sessão (tarefas de casa). Os estudos que investigaram os efeitos desta técnica relataram uma superioridade dela em relação ao placebo e à ausência de tratamento (Argyle, Bryant \& Trower, 1974; Falloon, Lindley \& McDonald, 1977). Comparações com outras técnicas de tratamento, não-farmacológicas, da fobia social não forneceram resultados consistentes (Shaw, 1979; Van Dam-Baggen \& Kraaimaat, 2000). O uso de técnicas de treinamento de habilidades sociais tem sido recomendado para todos os pacientes com fobia social, quer manifestem déficits de habilidades sociais quer não, pois este recurso tem se mostrado bastante eficaz em reduzir a ansiedade no confronto interpessoal (Curran, 1982; Heimberg, Juster, Hope \& Mattia, 1995; Lincoln \& cols., 2003).

\section{Exposição}

Exposição requer que o paciente imagine (exposição na imaginação) ou confronte realmente (exposição ao vivo) os estímulos temidos. O primeiro passo é construir junto com o paciente uma lista das situações temidas, do item que causa menos ansiedade ao que causa mais ansiedade e desconforto (Wolpe, 1973). As principais situações no tratamento da fobia social são falar diante de um grande público, comer e beber em público, conversar com outras pessoas, etc. (Lincoln \& cols., 2003; Mersch, 1995). Nos estágios inicias do tratamento com exposição, as situações são enfrentadas na companhia do terapeuta, até que possa ocorrer a habituação da ansiedade no item da hierarquia que está sendo confrontado. Após a exposição repetida e prolongada e quando a situação não eliciar mais altos níveis de ansiedade e desconforto, passa-se ao próximo item da lista de situações problemáticas. $\mathrm{O}$ processo continua até o paciente poder enfrentar todos os itens da hierarquia com significativa redução da ansiedade e do desconforto. Diversos estudos demonstraram a clara eficácia da exposição no tratamento da fobia social (Fava, Grand, Rafanelli, Conti \& Belluard, 2001; Heimberg, Dodge \& Hope, 1990; Lincoln \& cols., 2003; Mattick, Peters \& Clarke, 1989; Mattick \& Peters, 1988; Mersch, 1995; Newman, Hofmann, Trabert, Roth \& Taylor, 1994; Shaw, 1979).

\section{Reestruturação cognitiva}

A reestruturação cognitiva consiste em uma série de intervenções que se originaram das teorias e terapias cognitivas de Beck, Emery e Greenberg (1985) e Ellis (1962). Os pacientes com fobia social são ensinados a identificar estes pensamentos, fazer o teste da realidade e corrigir os conteúdos distorcidos e as crenças disfuncionais subjacentes. Esta reavaliação e correção das cognições distorcidas permitem ao paciente perceber que na grande maioria das vezes estava hipervalorizando negativamente uma situação e desvalorizando sua capacidade de enfrentamento da mesma situação (Clark \& Wells, 1995; Lucock \& Salkovskis, 1988; Taylor \& cols., 1997).

Segundo Beck, Emery e Greenberg (1985); Clark e Wells (1995); Lincoln e cols. (2003); Lucock e Salkovskis (1988) e Taylor e cols. (1997), basicamente as estratégias de reestruturação cognitiva objetivam ensinar ao paciente as seguintes modificações: observação e controle dos pensamentos irracionais e negativos; exame das evidências favoráveis e contrárias aos pensamentos distorcidos; e correção das interpretações tendenciosas por interpretações calcadas na realidade, o que geralmente resulta em redução sintomática da fobia social.

A técnica de reestruturação cognitiva procura desafiar diretamente as crenças irracionais ou disfuncionais, modificando-as ou substituindo-as por outras mais adaptativas. A técnica é aplicada tanto nos pensamentos que ocorrem antes da situação social como naqueles que ocorrem durante e após a situação temida (Clark \& Wells, 1995; Taylor \& cols., 1997).

Nesta abordagem de tratamento, as técnicas de exposição servem muito mais para eliciar as cognições negativas do que para habituar à ansiedade gerada pela situação social (Heimberg, 2001). Diversos estudos demonstraram que a reestruturação cognitiva é uma técnica eficaz no tratamento da fobia social, principalmente se for aplicada conjuntamente com técnicas de exposição (Lincoln \& cols., 2003; Lucock \& Salkovskis, 1988; Mattick, Peters \& Clarke, 1989; Mattick \& Peters, 1988; McManus, Clark \& 
Hackmann, 2000; Taylor \& cols., 1997; Turner, Beidel \& Cooley, 1994).

\section{STATUS ATUAL DA TERAPIA COGNITIVO- COMPORTAMENTAL NA FOBIA SOCIAL}

$\mathrm{Na}$ área da fobia social uma série de estudos de metaanálise reportaram uma alta eficácia da terapia cognitivo-comportamental na redução da ansiedade social (Feske \& Chambless, 1995; Gould, Buckminster, Pollack, Otto \& Yap, 1997; Heimberg, 2001; Otto, 1999; Taylor, 1996). Por exemplo, a metaanálise realizada por Feske e Chambless (1995) se concentrou na comparação de estudos que testaram a terapia cognitivo-comportamental e estudos que testaram somente a exposição no tratamento da fobia social. Os resultados apontaram que ambas as modalidades de tratamentos são eficazes, com uma ligeira superioridade da terapia cognitivo-comportamental sobre a técnica de exposição usada isoladamente. Taylor (1996), em seu estudo de metaanálise, comparou controles na lista de espera, placebo, exposição, reestruturação cognitiva, uma combinação de exposição e reestruturação cognitiva e treinamento de habilidades sociais no tratamento da fobia social. Nos resultados o autor encontrou que todos os tratamentos incluindo o placebo foram superiores aos controles na lista de espera que não estavam recebendo nenhum tratamento. Quando se excluíram os controles na lista de espera, o placebo foi a forma de tratamento que menos trouxe melhoras aos pacientes. A combinação de exposição e reestruturação cognitiva foi o tratamento que trouxe os benefícios mais significativos aos pacientes.

A abordagem em grupo da terapia cognitivocomportamental vem recebendo uma grande atenção dos pesquisadores, pois além das eficazes técnicas cognitivas e comportamentais da própria terapia, o fator grupo vem a contribuir em muito com os pacientes com fobia social, pois desta maneira diminui $o$ isolamento destes pacientes e age como uma forma de exposição (Heimberg, Juster, Hope \& Mattia, 1995; Heimberg, Salzman \& Holt, 1993; Hofmann, 2004).

Em relação à combinação da terapia cognitivocomportamental e da farmacoterapia no tratamento da fobia social, os autores Gelernter, Uhde e Cimbolic (1991); Heimberg, Liebowitz, Hope, Scheiner, Holt, Welkowitz, Juster, Campeas, Bruch, Cloitre, Fallon \& Klein (1998); Solyom, Solyom, LaPierre, Pecknold e Morton (1981) relataram que o acréscimo da farmacologia é de extrema valia ao tratamento cognitivo-comportamental, pois os medicamentos, principalmente os antidepressivos, facilitam a aplicação das técnicas comportamentais.

\section{CONSIDERAÇÕES FINAIS}

A fobia social é um dos mais prevalentes transtornos psicológicos na população geral. Seu correto diagnóstico e encaminhamento para tratamento adequado minimizam, se não todos, pelo menos os principais efeitos negativos que esta grave condição de saúde mental impõe a seus portadores. A terapia cognitivo-comportamental e a farmacologia, segundo a literatura científica, constituem-se hoje como as principais intervenções terapêuticas para os quadros de fobia social.

\section{REFERÊNCIAS}

APA (2002, 4 $4^{\text {a }}$ ed. rev.). DSM-IV-TR: manual diagnóstico e estatístico de transtornos mentais. Porto Alegre: Artmed.

Argyle, M., Bryant, B. M. \& Trower, P. (1974). Social skills training and psychoterapy: A comparative study. Psychological Medicine, 4(2), 435-443.

Beck, A. T., Emery, G. \& Greenberg, R. L. (1985). Anxiety disorders and phobias: A cognitive perspective. New York: Basic Books.

Clark, D. M. \& Wells, A. A. (1995). Cognitive model of social phobia. In R. G. Heimberg, M. R. Liebowitz, D. A. Hope \& F. R. Schneider. Social phobia: Diagnosis, assessment and treatment. (pp. 69-93). New York: Guilford.

Curran, J. P. (1982). A procedure for the assessment of social skills: The simulated social interaction test. In J. P. Curran. Social skills training: A practical handbook for assessment and treatment (pp. 116-132). New York: Guilford.

D’El Rey, G. J. F. (2001). Fobia social: mais do que uma simples timidez. Arquivos de Ciências da Saúde da Unipar, 5(3), 273-276.

Ellis, A. (1962). Irrational ideas that cause and maintain neuroses. In A. Ellis. Reason and emotion in psychotherapy (pp. 78-94). New York: Stuart.

Falloon, I. R. H., Lindley, P. \& McDonald, R. (1977). Social skills training of out-patient groups: A controlled study with rehearsal and home-work. British Journal of Psychiatry, 131(5), 599-609.

Fava, G. A., Grand, S., Rafanelli, C., Conti, S. \& Belluard, P. (2001). Long-term outcome of social phobia treated by exposure. Psychological Medicine, 31(5), 899-905.

Feske, U. \& Chambless, D. L. (1995). Cognitive-behavioral versus exposure only treatment for social phobia: A metaanalysis. Behavior Therapy, 26(6), 695-720.

Furmark, T., Tillfors, M., Stattin, H., Ekselius, L. \& Fredrikson, M. (2000). Social phobia subtypes in the general population revealed by cluster analysis. Psychological Medicine, 30(6), 1335-1344. 
Gelernter, C. S., Uhde, T. W. \& Cimbolic, P. (1991). Cognitive-behavioral and pharmacological treatments of social phobia. Archives of General Psychiatry, 49(9), 938945.

Gould, R. A., Buckminster, A., Pollack, P. M., Otto, M. W. \& Yap, R. T. (1997). Cognitive-behavioral and pharmacological treatment for social phobia: A metaanalysis. Clinical Psychology: Science and Practice, 4(2), 296-306.

Heimberg, R. G. (2001). Current status of psychotherapeutic interventions for social phobia. Journal of Clinical Psychiatry, 62(1), 36-42.

Heimberg, R. G., Dodge, C. S. \& Hope, D. A. (1990). Cognitive-behavioral treatment of social phobia: Comparison to a credible placebo control. Cognitive Therapy and Research, 14(1), 1-23.

Heimberg, R. G., Juster, H. R., Hope, D. A. \& Mattia, J. I. (1995). Cognitive-behavioral group treatment: Description, case presentation, and empirical support. In M. B. Stein. Social phobia: Clinical and research perspectives. (pp. 293-321). Whashington, DC: American Psychiatric.

Heimberg, R. G., Liebowitz, M. R., Hope, D. A., Scheiner, F. R., Holt, C. S., Welkowitz, L. A., Juster, H. R., Campeas, R., Bruch, M. A., Cloitre, M., Fallon, B. \& Klein, D. F. (1998). Cognitive-behavioral group therapy and phenelzine therapy for social phobia. Archives of General Psychiatry, 55(11), 1133-1141.

Heimberg, R. G., Salzman, D. G. \& Holt, C. S. (1993). Cognitive-behavioral group treatment for social phobia: Effectiviness of five year follow-up. Cognitive Research and Therapy, 17(3), 325-339.

Hofmann, S. G. (2004). Cognitive mediation of treatment change in social phobia. Journal of Consulting and Clinical Psychology, 72(3), 392-399.

Hofmann, S. G., Ehlers, A. \& Roth, W. (1995). Conditioning theory: A model for the etiology of publick speaking anxiety? Behaviour Research and Therapy, 33(4), 567571.

Jerremalm, A., Jansson, L. \& Öst, L. G. (1986). Cognitive and physiological reactivity and the effects of different behavioral methods in the treatment of social phobia. Behaviour Research and Therapy, 24(2), 171-180.

Kessler, R. C., McGonagle, K. A., Zhao, S., Nelson, C. B., Hughes, M., Eshleman, S., Wittchen, H-U. \& Kendler, K. S. (1994). Lifetime and 12-month prevalence of DSM-III-R psychiatric disorders in the United States: Results from the national comorbidity survey. Archives of General Psychiatry, 5l(1), 8-19.

Lamberg, L. (1998). Social phobia: Not just another name for shyness. Journal of American Medical Academy, 280(8), 685-686.

Liebowitz, M. R. (1999). Update the diagnosis and treatment of social anxiety disorder. Journal of Clinical Psychiatry, 60(suppl 18), 22-26.

Lincoln, T. M., Rief, W., Hahlweg, K., Frank, M., Schroeber, B. \& Fiegenbaum, W. (2003). Effectiveness of an empirically supported treatment for social phobia in the field. Behaviour Research and Therapy, 41(11), 12511269.

Lucock, M. P. \& Salkovskis, P. M. (1988). Cognitive factors in social anxiety and its treatment. Behaviour Research and Therapy, 26(4), 297-302.

Magee, W. J., Eaton, W. W., Wittchen, H-U., McGonagle, K. A. \& Kessler, R. C. (1996). Agoraphobia, simple phobia and social phobia in the national comorbidity survey. Archives of General Psychiatry, 53(2), 159-168.

Mattick, R. P. \& Peters, L. (1988). Treatment of severe social phobia: Effects of guided exposure with and without cognitive restructuring. Journal of Consulting and Clinical Psychology, 56(2), 251-260.

Mattick, R. P., Peters, L. \& Clarke, J. C. (1989). Exposure and cognitive restructuring for social phobia: A controlled study. Behavior Therapy, 20(1), 3-23.

McManus, F., Clark, D. M. \& Hackmann, A. (2000). Specificity of cognitive bases in social phobia and their role in recorery. Behavioural and Cognitive Psychotherapy, 28(3), 201-209.

Mersch, P. P. A. (1995). The treatment of social phobia: The differential effectiviness of exposure in vivo and an integration of exposure in vivo, rational emotive therapy and social skills training. Behaviour Research and Therapy, 33(2), 259-269.

Newman, M. G., Hofmann, S. G., Trabert, W., Roth, W. \& Taylor, S. (1994). Does behavioral treatment of social phobia lead to cognitive changes? Behaviour Research and Therapy, 25(5), 503-517.

Öst, L. G. \& Hugdahl, K. (1981). Acquisition of phobias and anxiety responses patterns in clinical patients. Behaviour Research and Therapy, 16(3), 439-447.

Öst, L. G., Jerremalm, A. \& Johansson, J. (1981). Individual responses patterns and the effect of different behavioral methods in the treatment of social phobia. Behaviour Research and Therapy, 19(1), 1-16.

Otto, M. W. (1999). Cognitive-behavioral therapy for social anxiety disorder: Model, methods and outcome. Journal of Clinical Psychiatry, 60(suppl 9), 14-19.

Schneider, F. R. (1995). Monoamine oxidase inhibitors, seletive sototonin reuptake inhibitors, and other antidepressants in pharmacotherapy. In M. B. Stein. Social phobia: Clinical and research perspectives (pp. 138-153). Washington: American Psychiatric.

Shaw, P. (1979). A comparison of three behaviour therapies in the treatment of social phobia. British Journal of Psychiatry, 134(6), 620-623.

Solyom, C., Solyom, L., LaPierre, Y., Pecknold, J. \& Morton, L. (1981). Phenelzine and exposure in the treatment of phobia. Biological Psychiatry, 16(2), 239-247.

Stein, M. B., Torgrud, L. J. \& Walker, J. R. (2000). Social phobia symptoms, subtypes and severity. Archives of General Psychiatry, 57(9), 1046-1052.

Stopa, L. \& Clark, D. M. (1993). Cognitive process in social phobia. Behaviour Research and Therapy, 31(2), 255-267. 
Taylor, S. (1996). Meta-analysis of cognitive-behavioral treatments for social phobia. Journal of Behavior Therapy \& Experimental Psychiatry, 27(1), 1-9.

Taylor, S., Woody, S., Koch, W. J., McLean, P., Patterson, R. J. \& Anderson, K. W. (1997). Cognitive restructuring in the treatment of social phobia: Efficacy and mode of action. Behaviour Modification, 21(4), 487-511.

Turner, S. M., Beidel, D. \& Cooley, M. R. (1994). A multicomponent of behavioral treatment for social phobia: Social effectiveness therapy. Behaviour Research and Therapy, 32(3), 381-390.
Van Dam-Baggen, R. \& Kraaimaat, F. (2000). Group social skills training or cognitive group therapy as the clinical treatment of choice for generalized social phobia? Journal of Anxiety Disorders, 14(5), 437-451.

Wolpe, J. (1973, 2 $2^{\mathrm{a}}$ ed.). The practice of behavior therapy. London: Pergamon.

Recebido em 05/04/2005

Aceito em 27/12/2005

Endereço para correspondência: Gustavo D’El Rey: Rua Bom Jesus, 274-B, CEP 03344-000, São Paulo-SP.

E-mail: g.delrey@bol.com.br 